\title{
AVALIAÇÃO DE ANGUSTIFOLIADICIDAS NA CULTURA DA SOJA EM MINAS GERAIS
}

\author{
ITAMAR FERREIRA DE SOUZA ${ }^{1}$ \\ ANTONIO MACHADO REZENDE ${ }^{1}$ \\ JULIO PEDRO LACA BUENDIA ${ }^{2}$ \\ MILTON YOSHIURA ${ }^{3}$ \\ ${ }^{1}$ Pesquisador EPAMIG -Caiixa Postal 176 - \\ Lavras-MG. \\ ${ }^{2}$ Pesquisador EPAMIG - Av. Amazonas, \\ 115 - Belo Horizonte-MG. \\ ${ }^{3}$ Pesquisador COTIA - Rodovia MG-235, \\ Km 1, São Gotardo-MG.
}

\section{RESUMO}

Três experimentos de campo foram conduzidos em Latossolos Vermelho-Escuro e Vermelho-Amarelo, nos anos 1981/82 e 1982/83 com o objetivo de determinar o efeito de herbicidas para o controle de plantas daninhas angustifoliadas e fitoto-xicidade sobre a cultura da so ja, cultivares UFV-1 e Cristalina. O grupo das acetanilidas e pendimethalin controlaram a trapoe raba. Para as três espécies latifoliadas, o acetochlor, trifluralin e oryzalin foram eficientes. Além disso, o metolachlor controlou a poaia e o pendimethalin controlou a poaia e o apaga-fogo. Para o controle do capimmarmelada, todos os produtos foram eficientes, exceto quizalofop-etil e mefluidide, enquanto que para o capim-colchão apenas o mefluidide não foi eficiente. Finalmente, o timbete não foi eficientemente controlado por alachlor, metolachlor, pentimethalin e mefluidide. Aceto- chlor e oryzalin afetaram negativamente o "stand" inicial. Alémdisso, o acetochlor reduziu altura da inserção da primeira vagem. O quizalofop-etil causou uma redução na produçao de grãos.

PALAVRAS CHAVE: augustifoliadas, acetanilidas, pendimethalin, acetochlor, trifluralin, oryzalin, metolachlor, poaia, apaga-fogo, marmelada, quizalofop-etil, sethoxydin.

\section{SUMMARY}

GRASS WEED CONTROL WITH HERBICIDES IN SOYBEANS IN MINAS GERAIS

Three field experiments were carried out on Dark Red Latosol and Yellow Red Latosol in 1981/82 and 1982/83 to evaluate the efficiency of herbicides upon grassy and their phytotoxicity upon "UFV - 1" and "Cristalina" soybeans cultivars. The acetoanilide group and pendi- 
methalin showed good spiderwort con trol. For the three broadleaved weed species, acetochlor, trifluralin and oryzalin were efficient. Moreover, metolachlor controlled Brazil pusley and pendimethalin controlled Brazil pusley and A. ficoidea. For alexandergrass control all herbicides tested were efficient but quizalofop-ethyl and mefluidide, whereas crabgrass was not controlled by mefluidide only. Acetochlor and oryzalin treatments de creased initial stand. Besides, acetochlor decreased height of insertion of first pod. Quizalofop - ethil reduced grain yield of soy beans.

KEY WORDS: acetoanilides, pendimethalin, acetochlor, trifluralin, oryzalin, metolachlor, Brazil pusley, A. ficoidea, alexandergrass, quizalofop ethil, mefluidide, sethoxydin.

\section{INTRODUÇÃO}

O controle das diferentes espécies de plantas daninhas monocotiledôneas e dicotiledôneas infestantes da cultura da soja cultivada sob condições de cerrado, tem sido motivo de preocupação da pesquisa. Em trabalhos de levantamento das condições agronômicas das lavouras de soja (Glycine $\max ($ L.) Merril) no Estado de Minas Gerais, realizado em 1979/80 por Arantes e Rezende (1981), observou-se que, das lavouras amostradas, $60 \%$ estavam praticamente livres de plantas daninhas mas, a infestação tendia a aumentar com os anos de cultivo. Em 1982/83 Souza et al. (1983) observaram nove espécies da família Gramineae, cinco Compositae, três Rubiaceae, duas Labiatae, duas Ama- ranthaceae, e uma espécie de cada das seguintes famílias: Commelinaceae, Convolvulaceae, Portulaca-ceae, Cruciferae, Malvaceae e Sola naceae.

As indicações de herbicidas para a soja em Minas Gerais tem sido baseadas em poucos resultados de pesquisas obtidos em anos anteriores ou naquelas indicações para ou trás regiões do país. Segundo Maia et al. (1977), várias espécies de plantas daninhas de folhas estreitas foram eficientemente controladas com a mistura de trifluralin ( $\alpha, \alpha, \alpha$-trifluoro - 2,6 - dinitro-N-N-dipropil$\mathrm{p}$ toluidina) e metribuzin [4-amino-6-tertbutil-3-(metiltio)-s-triazina-5 (4-ona] nas doses de 1,0 e 0,2 kg/ha. Deuber (1980) testando vários herbicidas para estudar a fitotoxicidade sobre a cultura da soja em solos argiloso e barrento, concluiu que o alachlor [2-cloro-2,6 - dietil-N (metoximetil) acetoalinida] foi o produto mais seletivo para a varie dade Santa Rosa. Pendimethalin[N(1-etilpropil) -3,4 dimetil-2,6 dinitrobenzenoamina] apresentou leves injúrias iniciais e trifluralin e vernolate (Spropil dipropiltiocarbamato) causaram injúrias mais acentuadas, chegando a reduzir a população em um dos três experimentos conduzidos. Paulo et al. (1980) concluíram também que mefluidide $\{\mathrm{N}$-[2,4-dimetil-5[trifluorometil) sulfonil] amino] fenilacetamida aplicado isoladamente apresentou sintomas de intoxicação 'as plantas de soja e Cerdeira \& Voll (1980) observaram fitotoxicidade com mefluidide e bentazon [3isopropil-1H - 2,1,3-benzotiadiazin-4 $(3 \mathrm{H})$ ona 2,2-dioxi do] na variedade Viçoja.

Es te trabalho teve como objetivos avaliar a eficiência de vários herbicidas recomendados para 
o controle de plantas daninhas an gustifoliadas infestantes da cultura da soja no Estado de Minas Gerais.

\section{MATERIAL E MÉTODOS}

Três experimentos de campo foram instalados, sendo dois na Estação Experimental do Centro Regional de Pesquisa do Triângulo e Alto Paranaíba (CRTP) em Uberaba, MG e um na Fazenda Experimental Rio Paranaíba (COTIA) em São Gotardo, MG, nos anos agrícolas 1981/82 e 1982/83, com os objetivos de se determinar a eficiência de herbicidas no controle das plantas daninhas angustifoliadas em soja, variedades UFV-1 e Cristalina.

Os experimentos foram instalados em Latossolo Vermelho Escuro (CRTP) e Latossolo Vermelho Amarelo (COTIA), cujas características, estão apresentadas no Quadro 1.

$\mathrm{O}$ delineamen to experimental foi $\mathrm{O}$ de blocos ao acaso com quatro repetições. Os tratamentos utiliza dos nos dois anos agrícolas estão apresentad os no Quadro 2.

Pa ra a ap li cação do s he rbici da s de pré-emergência utilizou-se um pulverizador costal pressuriza do à $\mathrm{CO}_{2}$ de dois bicos Te ej et 8003, a uma pressão de $40 \mathrm{psi}$, numa velocidade de $3 \mathrm{~km} / \mathrm{h}$, gastando-se 340 litros de calda por hectare. Para a aplicação de herbicidas de pós-emergência, utilizou-se um pulveri zador monociclo, munido de cinco bicos Hatsuta 80.03, numa pressão de 40 psi (50 psi para sethoxydin), a uma velocidade de $3 \mathrm{~km} / \mathrm{h}$, gastan do-se 340 litros/ha (400 litros pa ra sethoxydin).

As culturas foram plantadas num espaçamento de $50 \mathrm{~cm}$, em parcelas de 6 linhas, com $5 \mathrm{~m}$ de comprimen to. As aplicações dos herbicidas de pós-emergência foram feitas com a cultura no estádio de 3-4 trifólios e as plantas daninhas com 4 perfilhos.

As espécies das principais gramineas infestantes das áreas foram timbete [Cenchrus echinatis (L.)] , capim pé-degalinha [Eleusine indica (L.) Gaertn] capimcolchão (Digitaria horizontalis Willd.) e trapoeraba (Commelina sp), no CRTP e capim-marmelada [Branqchiaria plantaginea (Link) Hitch], na COTIA.

Em 1981/82 foi também avaliada a eficiência dos tratamentos sobre o controle de algumas espécies latifoliadas a saber: mentrasto (Ageratum conyzoides L.), poaia (Richardia brasiliensis Gomes) e apagafogo[Alternanthera ficoidea(L.) R. Br ].

Quadro 1. Resultados de anälises de solos obtidos das āreas do experimen to de angustifoliadicidas em soja. Uberaba e São Gortardo. $1981 / 82 / 83$.

\begin{tabular}{|c|c|c|c|}
\hline & \multicolumn{2}{|c|}{ Uberaba } & \multirow{2}{*}{$\begin{array}{c}\text { Säo Gotardo } \\
1982 / 83\end{array}$} \\
\hline & $1981 / 82$ & $1982 / 83$ & \\
\hline $\begin{array}{l}\text { Argila (\%) } \\
\text { M.0. (\%) } \\
\text { pH }\end{array}$ & $\begin{array}{r}21,0 \\
1,9 \\
6,1\end{array}$ & $\begin{array}{r}21,0 \\
1,2 \\
6,1\end{array}$ & $\begin{array}{r}31,0 \\
2,9 \\
6,2\end{array}$ \\
\hline
\end{tabular}


Quadro 2. Tratamentos usados no experimento de Angustifoliadicidas na cultura de soja em Minas Gerais.

\begin{tabular}{|c|c|c|c|c|}
\hline Name Camuml & Nome Comercial & $\begin{array}{l}\text { Formulação }{ }^{2} \text { e } \\
\text { Concentração }(\mathrm{g} / \ell)\end{array}$ & $\begin{array}{l}\text { Epoca de } \\
\text { Aplicacääo }\end{array}$ & $\begin{array}{c}\text { Dose 1.a. } / \mathrm{ha}^{4} \\
\text { (g ou ml) }\end{array}$ \\
\hline & \multicolumn{2}{|c|}{ Uberaha 1981/82 } & & \\
\hline Alachlor & Laço CE & CE 480 & PRE & 1440 \\
\hline Acetochlor & Fist & CE 960 & PRE & 1800 \\
\hline Metolachlor & Dual $720 \mathrm{DC}$ & CE 720 & PRE & 2160 \\
\hline Pendimethalin & Herbadcx $500 \mathrm{E}$ & CE 500 & PRE & 1100 \\
\hline Alachlor N & - & CE 480 & PRE & 1440 \\
\hline Oryzalin & Surflan 480 & SC 480 & PRE & 1120 \\
\hline Mefluidide & Embark + Agral 90;0,18 & PM 240 & POS & 380 \\
\hline Fluazifop-buttil & Fuzillade + Agral $90 ; 0,18$ & CE 250 & POS & 380 \\
\hline Diclofop-metil & Iloxan $28 \mathrm{BC}$ & CE 284 & POS & 850 \\
\hline Sethoxydin & Poast + Triona B; 2,08 & CE 184 & POS & 270 \\
\hline Trifluralin & Marcap CE & CE 445 & PPI & 890 \\
\hline Test. sem cap. & - & - & - & - \\
\hline Test. com cap. & thanghos & $=-$ & - & - \\
\hline & Uberaba e & ão Gotardo 1982/83 & & \\
\hline $\begin{array}{l}\text { Alachlor } \\
\text { Acetochlor }\end{array}$ & $\begin{array}{l}\text { Laco CE } \\
\text { Fist }\end{array}$ & & $\begin{array}{l}\text { PRE } \\
\text { PRE }\end{array}$ & $\begin{array}{l}2900 \\
1200\end{array}$ \\
\hline $\begin{array}{l}\text { Acetochlor } \\
\text { Metolachlor }\end{array}$ & Dual $720 \mathrm{BC}$ & CE 720 & PRE & 2160 \\
\hline Pendimethalin & Herbadox $500 \mathrm{E}$ & CE 500 & PRE & 1100 \\
\hline Mefluidide & Embark + Agral 90;0,18 & PM 240 & POS. & 380 \\
\hline Fluazifop-but11 & Fusilade + Agral 90;0,18 & CE 250 & POS & 380 \\
\hline Diclofop-metil & Iloxan $28 \mathrm{BC}$ & CE 284 & POS & 850 \\
\hline Sethoxydin & Poast + Assist; $1,5 \mathrm{\ell} / \mathrm{ha}$ & CE 184 & POS & 230 \\
\hline Chlorazifop-propinil & Topik + Agral 90; 0,258 & CE 250 & POS & 250 \\
\hline Alloxydin-sodio & $\begin{array}{l}\text { Grasmat } 750 \text { PS+Trional B; } \\
2,08\end{array}$ & PS 750 & POS & 1120 \\
\hline Quizalofop-etil & Assure & CE 96 & POS & 70 \\
\hline Trifluralin & Marcap CE & CE 445 & PPI & 890 \\
\hline Test. sem cap. & - & - & - & - \\
\hline Test. can cap. & - & - & - & - \\
\hline
\end{tabular}

Test. com cap.

1. Names comuns de surfactantes nào foram citados devido as complexidades de padronizaçáo destes names.

2. $\mathrm{CE}=$ concentrado emulsionável; $\mathrm{SC}=$ solucāo concentrada, $\mathrm{PM}=\mathrm{pó}$ molhável; $\mathrm{PS}=\mathrm{pó}$ solúvel.

3. $\mathrm{PRE}=$ =pré-emergêncla; $\mathrm{POS}=\mathrm{pös-emergência;} \mathrm{PPI=prë-plantio} \mathrm{incorporado.}$

4. i.a.=inqrediente ativo. 
Os dados de precipitação pluviométrica dos dois locais durante a condução dos experimentos encontram-se no Quadro 3.

Os parâmetros utilizados para a avaliação dos tratamentos foram percentagem visual de controle de plantas daninhas, "stand" inicial, altura de plantas de soja, altura de inserção da primeira vagem, "stand" final e produção de grãos. Para a comparação das médias, utilizou-se os testes Tukey e Duncan ao nível de 5\% de probtabilidade.

\section{RESULTADOS E DISCUSSÃO}

As percentagens visuais de controle de plantas daninhas em Uberaba, enc ontram-se nos Quadros 4, 5 e 6.

Para o controle da trapoeraba (Quadro 4), o grupo das ace to-anilidas mostrou-se eficiente. Também o ace tochlor con trolou satisfatoriamente o mentrasto, a poaia e apaga-fogo e o metolachl or controlou a poaia (Quadro 6). $\mathrm{O}$ pendimethalin apresentou um controle apenas razoável para as angustifoliadas no ano 1982/83 sendo ainda menos eficiente para timbete (Quadro 5). Por outro lado, esse produto apresentou um controle satisfatório para trapoeraba (Quadro 4), poaia e apaga-fogo (Quadro 6). Ao contrário, o trifluralin não foi eficiente par a a trapoeraba con trolan do no entanto, todas as espécies estudadas. Como último dos três produtos das dinitroanilinas,o oryzal in se comportou semelhantemente ao trifluralin, deixando de controlar a trapoeraba e apresentando ótimo controle para as demais espécies est uda das .

Os produtos testados em pósemergência não apresentaram comtrole satisfatório para as lafito - liadas em estudo (Quadro 6) nem para trapoeraba (Quadro 4). O mefluidide em especial mostrou algum controle somente para o capim pé-de-gali nha no ano 82/83.

Os resultados de controle de marmelada, "stands", altura de inserção da primeira vagem, altura de plantas e produção de grãos estão apresentados nos Quadros 7, 8 e 9.

O "stand" inicial foi estatisticamente reduzido pelos tratamentos com acetochlor e oryzalin (Quadro 7), sendo que este último apresentou também o menor "stand" final e o primeiro, a mais baix a percentagem de emergência (Quadro 8). A altura de inserção da primeira vagem foi significativamente reduzida por dois anos cons ecutivos pelo aceto chlor sendo também o único tratamento estatisticamente inferior à testemunha capinada para a altura de plantas em Uberaba (Quadro 8).

Para produção de grãos, observou-se que $\mathrm{o}$ tratamento com metolachlor apresentou valores mais altos sendo que no ano 1981/82 (Quadro 7) foi o único tratamento estatisticamente superior à teste munha sem capina, além da testemunha com capina. O quizalofop-etil apesar de testado apenas um ano, não apresentou resultados satisfatórios para produção quando comparados com os outros tratamentos. Observou-se pelo Quadro 9 que este tratamento foi o que apresentou menor controle de capim marmelada podendo ter sido esta a razão da baixa produção apresentada por este tratamento. $\mathrm{O}$ mefluidide também deix ou a desejar no que diz respeito ao controle desta espécie.

\section{LITERATURA CITADA}

Arantes, N.E. \& Resende, A.M. de. 
Quadro 3. Precipitaçāo pluviométrica diāria, em mm, observadas no perío do de condução do experimento angustifoliadicidas em soja.

Uberaba e Sao Gotardo. Anos Agrícolas 1981/82 e 1982/83.

\begin{tabular}{|c|c|c|c|c|c|c|c|c|c|}
\hline & \multicolumn{6}{|c|}{ UBERABA } & \multicolumn{3}{|c|}{ SÃO GOTARDO } \\
\hline & Nov 81 & Dez 81 & $\operatorname{Jan} 82$ & Nov 82 & Dez 82 & $\operatorname{Jan} 83$ & Nov 82 & Dez 82 & $\operatorname{Jan} 83$ \\
\hline 1 & 0,0 & 25,4 & 0,0 & 0,0 & 16,0 & 0,4 & 0,0 & 0,0 & 18,0 \\
\hline 2 & 1,0 & 38,6 & 6,7 & 0,0 & 0,5 & 1,8 & 0,0 & 12,5 & 27,0 \\
\hline 3 & 1,0 & 14,5 & 87,6 & 0,0 & 45,2 & 4,1 & 0,0 & 39,5 & 2,5 \\
\hline 4 & 8,2 & 31,8 & 31,2 & 0,0 & 35,0 & 0,2 & 0,0 & 0,0 & 37,0 \\
\hline 5 & 8,4 & 0,2 & 2,4 & 0,0 & 0,0 & 8,0 & 0,0 & 0,0 & 27,5 \\
\hline 6 & 0,0 & 1,0 & 0,0 & 0,3 & 2,6 & 6,6 & 0,0 & 17,5 & 45,0 \\
\hline 7 & 0,0 & 16,5 & 2,0 & 2,4 & 42,4 & 9,6 & 0,0 & 0,0 & 1,5 \\
\hline 8 & 78,8 & 16,9 & 0,0 & 0,0 & 6,4 & 0,0 & 17,5 & 2,5 & 10,0 \\
\hline 9 & 13,2 & 12,9 & 1,2 & 0,0 & 0,0 & 1,2 & 0,0 & 36,0 & 15,0 \\
\hline 10 & 32,6 & 61,5 & 3,2 & 2,8 & 5,6 & 8,6 & 0,0 & 0,0 & 0,0 \\
\hline $\mathbf{1 1}$ & 0,0 & 1,6 & 5,3 & 75,0 & 18,0 & 17,7 & 0,0 & 0,0 & 0,0 \\
\hline 12 & 10,0 & 13,4 & 65,7 & 14,1 & 6,2 & 4,4 & 0,0 & 0,0 & 0,0 \\
\hline 13 & 1,0 & 0,0 & 6,2 & 0,0 & 15,1 & 4,0 & 0,0 & 0,0 & 44,5 \\
\hline 14 & 10,6 & 0,0 & 0,0 & 0,0 & 18,2 & 9,4 & 12,5 & 0,0 & 0,0 \\
\hline 15 & 0,4 & 0,0 & 0,0 & 41,9 & 0,4 & 8,3 & 30,0 & 5,0 & 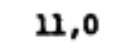 \\
\hline 16 & 0,0 & 23,2 & 0,4 & 29,7 & 12,3 & 17,0 & 0,0 & 3,0 & 2,5 \\
\hline 17 & 0,0 & 10,8 & 0,0 & 0,0 & 14,0 & 10,7 & 0,0 & 1,0 & 6,5 \\
\hline 18 & 0,0 & 11,2 & 0,0 & 4,7 & 18,3 & 15,7 & 0,0 & 0,0 & 88,0 \\
\hline 19 & 0,0 & 1,2 & 0,0 & 0,0 & 1,0 & 53,6 & 0,0 & 15,0 & 30,0 \\
\hline 20 & 6,0 & 0,0 & 0,0 & 3,3 & 17,6 & 21,0 & 1,0 & 0,0 & 0,0 \\
\hline 21 & 4,4 & 0,0 & 1,4 & 2,4 & 2,6 & 8,1 & 0,0 & 11,0 & 28,0 \\
\hline 22 & 0,0 & 2,6 & 0,0 & 5,4 & 23,4 & 0,0 & 0,0 & 0,0 & 0,0 \\
\hline 23 & 0,0 & 5,0 & 22,8 & 0,0 & 3,0 & 0,0 & 0,0 & 1,0 & 17,5 \\
\hline 24 & 0,0 & 3,3 & 16,0 & 22,8 & 21,1 & 2,4 & 0,0 & 52,0 & 0,0 \\
\hline 25 & 0,0 & 0,0 & 0,9 & 0,0 & 3,4 & 0,0 & 0,0 & 0,0 & 70,0 \\
\hline 26 & 0,0 & 0,0 & 8,8 & 0,0 & 0,0 & 39,1 & 0,0 & 0,0 & 38,0 \\
\hline 27 & 0,0 & 22,6 & 6,7 & 15,0 & 0,6 & 4,2 & 0,0 & 4,0 & 3,5 \\
\hline 28 & 0,0 & 35,6 & 12,4 & 0,0 & 37,1 & 11,4 & 11,0 & 7,5 & 3,0 \\
\hline 29 & 7,0 & 28,8 & 26,8 & 3,0 & 1,4 & 0,6 & 2,0 & 5,0 & 3,0 \\
\hline 30 & 1,0 & 0,0 & 19,8 & 0,0 & 19,8 & 0,0 & 0,0 & 86,0 & 0,0 \\
\hline 31 & - & 0,2 & 4,8 & - & 40,7 & 0,6 & - & 0,0 & 30,5 \\
\hline TOTA & L 183,6 & 396,8 & 326,0 & 222,8 & 427,9 & 308,3 & 74,0 & 298,5 & 559,5 \\
\hline
\end{tabular}




\begin{tabular}{|c|c|c|c|c|c|c|}
\hline \multirow{2}{*}{ Tratamentos } & \multicolumn{2}{|c|}{ Trapoeraba } & \multicolumn{2}{|c|}{$P \bar{e}-d e-g a l i n h a$} & \multicolumn{2}{|c|}{ Colchão } \\
\hline & 30 d.a.a. ${ }^{3}$ & 45 d.a.a. & 30 d.a.a. & 45 d.a.a. & 30 d.a.a. & 45 d.a.a. \\
\hline Alachlor & 100,0 & 100,0 & 54,5 & 38,5 & 52,0 & 50,8 \\
\hline Ace tochlor & 91,7 & 88,1 & 98,3 & 93,7 & 88,0 & 77,8 \\
\hline Metolachlor & 100,0 & 100,0 & 100,0 & 100,0 & 74,7 & 71,4 \\
\hline Pendime thal in & 76,1 & 77,4 & 96,6 & 89,7 & 88,0 & 92,1 \\
\hline Alachlor-N & 92,0 & 91,7 & 100,0 & 100,0 & 81,3 & 66,7 \\
\hline Oryzalin & 56,2 & 40,5 & 100,0 & 100,0 & 100,0 & 100,0 \\
\hline Mefluidide 1 & 59,2 & 61,9 & 50,6 & 60,3 & 0,0 & 0,0 \\
\hline Fluazifop-butill & 54,2 & 57,1 & 100,0 & 100,0 & 92,1 & 100,0 \\
\hline Diclofop-metill & 56,2 & 56,0 & 98,3 & 100,0 & 77,3 & 79,4 \\
\hline Sethoxydin ${ }^{2}$ & 52,2 & 53,6 & 96,6 & 100,0 & 100,0 & 100,0 \\
\hline Trifluralin & 18,4 & 0,0 & 96,0 & 94,8 & 100,0 & 100,0 \\
\hline $\begin{array}{l}\text { Test. sem capina } \\
\text { Test. com capina }\end{array}$ & $\begin{array}{r}0,0 \\
100,0\end{array}$ & $\begin{array}{r}0,0 \\
100,0\end{array}$ & $\begin{array}{r}0,0 \\
100,0\end{array}$ & $\begin{array}{r}0,0 \\
100,0\end{array}$ & $\begin{array}{r}0,0 \\
100,0\end{array}$ & $\begin{array}{r}0,0 \\
100,0\end{array}$ \\
\hline
\end{tabular}

$1=$ Agral 90 a $0,1 \%$ adicionado a calda de pulverização.

2 = Triona B a $2,0 \%$ adicionada à calda de pulverização.

$3=$ d.a.a. = dias após a aplicação do tratamento. 
Quadro 5. Percentagens de controle de angustifoliadas obtidas no experimento de angustifoliadicidas na cultura da soja. Uberaba, $1982 / 83$.

\begin{tabular}{|c|c|c|c|c|c|c|c|c|}
\hline \multirow[b]{2}{*}{ Tratamentos } & \multicolumn{2}{|c|}{ Timbete } & \multicolumn{3}{|c|}{ Pé-de-Galinha } & \multicolumn{3}{|c|}{ Colchão } \\
\hline & 45 d.a. $a^{5}$ & 60 d.a.a & 30 d.a.a & 45 d.a.a & 60 d.a.a & 30 d.a.a & 45 d.a.a & 60 d.a.a \\
\hline Alachlor & $70,0 b c^{6}$ & $48,7 c$ & $97,5 a b$ & $92,5 a b$ & $92,5 a b$ & $91,2 \mathrm{a}$ & 75,0 ode & 72,5 ode \\
\hline Acetochlor & $85,0 a b c$ & $86,2 a b$ & $96,2 a$ & $91,2 a b$ & $87,5 \mathrm{~b}$ & $95,0 \mathbf{a}$ & $92,5 \mathbf{a}$ & $88,7 a b$ \\
\hline Metolachlor & $63,7 \mathrm{c}$ & $58,7 \mathrm{c}$ & $96,2 a$ & $92,5 a b$ & $85,0 \mathrm{~b}$ & $85,0 a b$ & 77,5 ode & $82,5 \mathrm{bc}$ \\
\hline Pendimethalin & 15,0 de & $23,7 \quad d$ & $63,7 \mathrm{~b}$ & 71,2 d & $67,5 \mathrm{c}$ & $70,0 \mathrm{~b}$ & $63,7 \mathrm{e}$ & $78,7 \mathrm{bod}$ \\
\hline Mefluididel & $23,7 \mathrm{~d}$ & 21,2 d & $70,0 \mathrm{~b}$ & $77,5 \propto d$ & $87,5 \mathrm{~b}$ & $47,5 \mathrm{c}$ & 67,5 de & $65,0 \mathrm{~d}$ \\
\hline Fluazifop-butill & $83,7 a b c$ & $86,2 a b$ & $90,0 \mathrm{a}$ & $91,2 \mathrm{ab}$ & $90,0 a b$ & $82,5 \mathrm{ab}$ & $87,5 a b c$ & $88,7 a b$ \\
\hline Diclofop-metil & $83,7 a b c$ & $87,5 a b$ & $91,2 \mathrm{a}$ & $90,0 \mathrm{ab}$ & $90,0 \mathrm{ab}$ & $81,2 a b$ & 82,5 bod & $81,2 \mathrm{bc}$ \\
\hline Sethoxydin 2 & $86,2 \mathrm{ab}$ & $83,7 \mathrm{ab}$ & $90,0 \mathrm{a}$ & $87,5 b c$ & $92,5 a b$ & $86,2 a b$ & $88,7 a b c$ & $87,5 a b c$ \\
\hline Chlorazifop-propind1 ${ }^{3}$ & $90,0 \mathrm{ab}$ & $88,7 a b$ & $92,5 a$ & $92,5 a b$ & $92,5 a b$ & $92,5 a$ & $87,5 a b c$ & $87,5 \mathrm{ab}$ \\
\hline Alloxydin 4 & $85,0 a b c$ & $81,2 \mathrm{~b}$ & $90,0 a$ & $88,7 \mathrm{~b}$ & $87,5 \mathrm{~b}$ & $86,2 a b c$ & $86,2 a b c$ & $88,7 a b$ \\
\hline Quizalofop-etil & $86,2 \mathrm{ab}$ & $83,7 \mathrm{ab}$ & $92,5 a$ & $90,0 a b$ & $90,0 \mathrm{ab}$ & $92,5 \mathrm{ab}$ & $87,5 \mathbf{a}$ & $86,2 a b c$ \\
\hline Trifluralin & $85,0 a b c$ & $83,7 a b$ & $88,7 a$ & $92,5 a b$ & $86,2 \mathrm{~b}$ & $86,2 \mathrm{~b}$ & $88,7 a b c$ & 81,2 bc \\
\hline Test. sem capina & 0,0 e & $0,0 \mathrm{e}$ & $0,0 \mathrm{c}$ & $0,0 e$ & $0,0 \quad d$ & $0,0 \mathrm{~d}$ & $0,0 \mathrm{f}$ & $0,0 \mathrm{e}$ \\
\hline Test. com capina & $100,0 \mathrm{a}$ & $100,0 \mathrm{a}$ & $100,0 a$ & $100,0 a$ & $100,0 \mathrm{a}$ & $100,0 \mathrm{a}$ & $100,0 \mathrm{a}$ & $100,0 \mathrm{a}$ \\
\hline C.v (8) & 11,8 & 10,3 & 7,0 & 4,9 & 5,6 & 9,5 & 7,9 & 7,4 \\
\hline
\end{tabular}

I - Agral 90 a 0,1 adicionado à calda de pulverização

2 - Assist a 1,5 $\mathrm{l} / \mathrm{ha}$ adicionado à calda de pulverização

3 = Agral a 0,25 adicionado à calda de pulverização

4 - Triona B a 2,0 a dicionada à calda de pulverização

5 - d.a.a = dias apó a aplicação do tratamento

6 - As módias dentro da mesma coluna, seguidas pelas mesnas letras, não diferem estatisticamente entre si pelo teste de Tukey a nivel de 5 i de probabilidade. 


(

\begin{tabular}{|c|c|c|c|c|c|c|}
\hline \multirow{2}{*}{ Tratamentos } & \multicolumn{2}{|c|}{ Mentrasto } & \multicolumn{2}{|c|}{ Poaia } & \multicolumn{2}{|c|}{ Apaga-fogo } \\
\hline & $30 \mathrm{~d} \cdot \mathrm{a} \cdot \mathrm{a}^{3}$ & 45 d.a.a & $30 \mathrm{~d} \cdot \mathrm{a} \cdot \mathrm{a}$ & 45 d.a.a & $30 \mathrm{~d} \cdot \mathrm{a} \cdot \mathrm{a}$ & 45 d.a.a \\
\hline Alachlor & 73,7 & 64,6 & 48,6 & 66,7 & 73,0 & 75,6 \\
\hline Ace tochlor & 89,5 & 86,2 & 97,1 & 97,4 & 87,7 & 88,2 \\
\hline Metolachlor & 0,0 & 0,0 & 100,0 & 100,0 & 56,0 & 54,8 \\
\hline Pendimethalin & 0,0 & 0,0 & 74,3 & 74,4 & 91,6 & 97,1 \\
\hline Alachlor-N & 77,9 & 3,1 & 74,3 & 79,5 & 70,8 & 73,3 \\
\hline Oryzalin & 100,0 & 100,0 & 100,0 & 100,0 & 100,0 & 100,0 \\
\hline Mefluidide ${ }^{1}$ & 73,7 & 69,2 & 43,3 & 51,3 & 42,8 & 56,6 \\
\hline Fluazifop-buti $1^{1}$ & 51,6 & 0,0 & 0,0 & 35,9 & 13,8 & 31,9 \\
\hline Diclofop-metill & 0,0 & 0,0 & 65,7 & 59,0 & 19,9 & 16,5 \\
\hline Sethoxydin 2 & 35,8 & 27,7 & 28,6 & 23,1 & 0,0 & 2,3 \\
\hline Trifluralin & 29,5 & 87,7 & 100,0 & 100,0 & 100,0 & 100,0 \\
\hline Test. sem capina & 0,0 & 0,0 & 0,0 & 0,0 & 0,0 & 0,0 \\
\hline Test. com capina & 100,0 & 100,0 & 100,0 & 100,0 & 100,0 & 100,0 \\
\hline
\end{tabular}

1 = Agral 90 a $0,1 \%$ adicionado à calda de pulverização.

2 = Triona B a $2,0 \%$ adicionada à calda de pulverização.

3 = d.a.a = dias após aplicação do tratamento. 
Quadro 7. Resultados médios de "stands", altura de inserção da primeira vagem e produção de grãos obtidos no experimento de angustifoliadicidas na cultura da soja. Uberaba, 1981/82.

\begin{tabular}{|c|c|c|c|c|}
\hline Tratamentos & $\begin{array}{l}\text { "Stand" } \\
\text { inicial } \\
\left(\mathrm{pl} / \mathrm{m}^{2}\right)\end{array}$ & $\begin{array}{l}\text { "St and" } \\
\text { fina1 } \\
\left(\mathrm{p} 1 / \mathrm{m}^{2}\right)\end{array}$ & $\begin{array}{c}\text { Alt. Inserção } \\
\text { lạ vagem } \\
(\mathrm{cm})\end{array}$ & $\begin{array}{l}\text { Produção } \\
\text { (kg/ha) }\end{array}$ \\
\hline Alachlor & $45,67 a b^{3}$ & $37,80 \mathrm{abc}$ & 15,5 a & $1942 \mathrm{abcd}$ \\
\hline Ace toch1or & $40,21 \mathrm{bc}$ & $34,37 \mathrm{bcd}$ & $10,2 \mathrm{~d}$ & 1861 bcde \\
\hline Metolachlor & 48,86 a & 41,20 a & 15,5 a & $2263 \mathrm{ab}$ \\
\hline Pendimethalin & $42,43 \mathrm{ab}$ & $34,30 \mathrm{bcd}$ & 15,5 a & $1931 \mathrm{abcd}$ \\
\hline Alachlor-N & 48,96 a & $37,25 a b c$ & 16,5 a & $2101 \mathrm{abc}$ \\
\hline Oryzalin & $35,83 \mathrm{c}$ & $25,17 \mathrm{~d}$ & $12,8 \mathrm{bc}$ & 1802 bcde \\
\hline Mefluidide ${ }^{l}$ & 48,82 a & $34,58 \mathrm{bcd}$ & $14,8 \mathrm{ab}$ & $1403 \mathrm{e}$ \\
\hline Fluazifop-butil 1 & $46,25 \mathrm{ab}$ & $32,63 \mathrm{~cd}$ & 15,2 a & $1564 \mathrm{de}$ \\
\hline Diclofop-metil & 46,81 ab & 38,75 ac & $14,2 \mathrm{bc}$ & 1748 cde \\
\hline Sethoxydin 2 & 47,94 a & $33,20 \mathrm{~cd}$ & 16,0 a & $1581 \mathrm{de}$ \\
\hline Trifluralin & 47,06 a & $38,87 \mathrm{ab}$ & 15,8 a & 1786 bcde \\
\hline Test. sem capina & $43,22 a b$ & $31,20 \mathrm{~d}$ & 15,8 a & $1625 \mathrm{cde}$ \\
\hline Test. com capina & $46,14 \mathrm{ab}$ & $38,97 \mathrm{ab}$ & $12,2 \mathrm{~cd}$ & 2386 a \\
\hline
\end{tabular}

$1=$ Agral 90 à $0,1 \%$ adicionado à calda de pulverização.

2 = Triona $\mathrm{B}$ à $2,0 \%$ adicionada à calda de pulverização.

3 = As médias, dentro da mesma coluna, seguidas pelas mesmas letras, não diferem estatisticamen te entre si de acordo com o teste Duncan ao nivel de $5 \%$ de probabilidade. 
Condições Agronômicas das Lavou ras de Soja no Estado de Minas Gerais, $1979 / 80$. In: SEMINARIO NACIONAL DE PESQUISA DE SOJA. Anais. Fev. 1981. Vol . 1. EMBRAPA. Londrina, PR. p.139-152, 1982 .

Cerdeira, A.L. \& V0ll, E. Eficiência e fitotoxicidade de herbici das pós-emergência na cultura da soja (Glyxine max) para o controle de Gramíneas. In: CONGRESSO BRASILEIRO DE HERBICIDAS E ERVAS DANINHAS, XIII, Resumos, I1héus, Itabuna, BA. Jutho de 1980. p.59., 1980.

Deuber, R. Efeitos de Herbicidas e Densidade de Plantio no Desen volvimento e Produçäo da Sojă (Glycine max cv. Santa Rosa). In: CONGRESSO BRASILEIRO DE HER BICIDAS E ERVAS DANINHAS, XIII, Resumos. Ilheus, Itabuna, BA. Julho de 1980 , p.56, 1980.
Maia, A.C. \& Resende, A.M. Avaliação de Herbicidas na Cultura da Soja em Uberava e Rio Paranaiba /MG. Projeto Soja, Relatörio Anual 1977/79, EPAMIG, Be lo Horizonte. MG. p.60-65, 1982 .

Paulo, E.M.; Toledo, N.P.; Forster, R. Controle de Mono e Dicotile dôneas na Cultura da Soja em Pós-emergência pela combinação de Mefluidide e bentazon. In: CONGRESSO BRASILEIRO DE HERBICIDAS E ERVAS DANINHAS, XIII, Resumos, I lhéus, Itabuna, BA. Julho de 1980. p.577, 1980.

Souza, I.F.; Rafael, J.0.V.; A.M. Cunha, L.H.S. Estudos de Plantas Daninhas da Cultura da Soja (Glycine max (L) Merr.) em Minas Gerais. Relatório de Pesqui sa Projeto 005/014/7. EMBRAPA. Londrina. PR. $4 p$. 